\title{
Magnetic and structural investigation of growth induced magnetic anisotropies in $\mathrm{Fe}_{50} \mathrm{Co}_{50}$ thin films
}

\author{
M. Tamisari ${ }^{1, a}$, G. Ausanio ${ }^{2}$, V. Guidi ${ }^{1}$, V. Iannotti ${ }^{2}$, I. Neri ${ }^{1}$, F. Spizzo ${ }^{1}$, and P. Vavassori ${ }^{1,3}$ \\ 1 Department of Physics and CNISM, University of Ferrara, via G. Saragat 1, Building C I-44122 Ferrara, Italy \\ 2 CNR-SPIN and Dept. of Physical Sciences, "Federico II" University of Naples, p.le V. Tecchio 80 I-80125 Naples, Italy \\ 3 CIG nanoGUNE consolider, Tolosa Hiribidea, 76 E-20018 Donostia, San Sebastian, Spain
}

\begin{abstract}
In this paper, we investigate the magnetic properties of $\mathrm{Fe}_{50} \mathrm{Co}_{50}$ polycrystalline thin films, grown by dc-magnetron sputtering, with thickness $(t)$ ranging from $2.5 \mathrm{~nm}$ up to $100 \mathrm{~nm}$. We focused on the magnetic properties of the samples to highlight the effects of possible intrinsic stress that may develop during growth, and their dependence on film thickness. Indeed, during film deposition, due to the growth technique and growth conditions, a metallic film may display an intrinsic compressive or tensile stress. In our case, due to the $\mathrm{Fe}_{50} \mathrm{Co}_{50}$ magnetolastic properties, this stress may in its turn promote the development of magnetic anisotropies. Samples magnetic properties were monitored with a SQUID magnetometer and a magneto-optic Kerr effect apparatus, using both an in-plane and an out-of-plane magnetic field. Magnetoresistance measurements were collected, as well, to further investigate the magnetic behavior of the samples. Indications about the presence of intrinsic stress were obtained accessing samples curvature with an optical profilometer. For $t \leq 20 \mathrm{~nm}$, the shape of the in-plane magnetization loops is squared and coercivity increases with $t$, possibly due to fact that, for small $t$ values, the grain size grows with $t$. The magnetoresistive response is anisotropic in character. For $t>20 \mathrm{~nm}$, coercivity smoothly decreases, the approach to saturation gets slower and the shape of the whole loop gets less and less squared. The magnetoresistive effect becomes almost isotropic and its intensity increases of about one order of magnitude. These results suggest that the magnetization reorientation process changes for $t>20 \mathrm{~nm}$, and are in agreement with the progressive development of an out-of-plane easy axis. This hypothesis is substantiated by profilometric analysis that reveals the presence of an in-plane compressive stress.
\end{abstract}

\section{Introduction}

$\mathrm{Fe}_{50} \mathrm{Co}_{50}(\mathrm{FeCo})$ alloys are technologically important both for their high saturation magnetization and spin polarization factor [1] and for their possible application in magnetic devices, with perpendicular magnetization, as well [2]. However, FeCo is highly magnetostrictive and susceptible to stress-induced anisotropies. Indeed, the presence of compressive or tensile stress may result in an additional source of magnetic anisotropy, thus affecting the magnetic structure of the sample [3]. In particular, besides being induced by mechanical strain [4], the presence of an intrinsic stress may be favored by growth conditions [5]. In this contribution, we present the magnetic and magnetoresistive results obtained on $\mathrm{FeCo}$ polycrystalline thin films grown by dc-magnetron sputtering in Ar atmosphere as a function of FeCo thickness; these films were studied in view of their possible application in spin-valve or single-film devices that couple magnetoresistive and magnetostrictive properties. In more detail, we investigate their magnetic and magnetoresistive behavior to point out the possible effects of magnetic anisotropy due to intrinsic stress states.

\section{Experimental}

The FeCo layers were produced with a dc-magnetron sputtering apparatus using a $\mathrm{Fe}_{50} \mathrm{Co}_{50}$ composite target. They

\footnotetext{
a e-mail: tamisari@fe.infn.it
}

were deposited on naturally oxidated $\mathrm{Si}(100)$ substrates at room-temperature in a $1.3 \mathrm{~Pa} \mathrm{Ar}$ atmosphere with a sputtering rate equal to $0.02 \mathrm{~nm} / \mathrm{s}$. The substrates were rotated during growth to obtain a uniform thickness and the samples were covered with a $5 \mathrm{~nm}$ Cr overlayer to prevent them from oxidation. The FeCo films thickness ranges from $t=$ $2.5 \mathrm{~nm}$ up to $t=100 \mathrm{~nm}$. Magnetization $(M)$ measurements were performed at room temperature with a Quantum Design SQUID magnetometer. The data were collected with an external magnetic field $(H)$ applied both parallel and perpendicular to the samples surface. The magnetic properties of the samples were also investigated with a magnetooptic Kerr effect (MOKE) apparatus using the longitudinal MOKE configuration, to sense the $H$ dependence of both the $M$ in-plane components, the parallel to $H$ and the perpendicular to $H$ (transverse) one, and the polar configuration, to collect out-of-plane loops. Magnetoresistance (MR) data were collected at room temperature with the 4-probes method in the Van der Pauw configuration. The measurements were performed in different configurations, namely with the current both parallel and perpendicular to $H$, so to single out anisotropic and isotropic contributions to MR. The structural characterization was carried out in Bragg geometry $(\mathrm{Cu} \mathrm{K} \alpha$ radiation, $\lambda=0.154 \mathrm{~nm})$ with a high-resolution X-ray diffractometer (X'Pert Pro MRD XL PANalyticalTM). The change in substrate curvature induced by FeCo thin films deposition was measured using an optical profilometer (VEECOTM NT1100) with $1 \mu \mathrm{m}$ lateral and $1 \mathrm{~nm}$ vertical resolution. The profilometer is

This is an Open Access article distributed under the terms of the Creative Commons Attribution License 2.0, which permits unrestricted use, distribution, and reproduction in any medium, provided the original work is properly cited. 


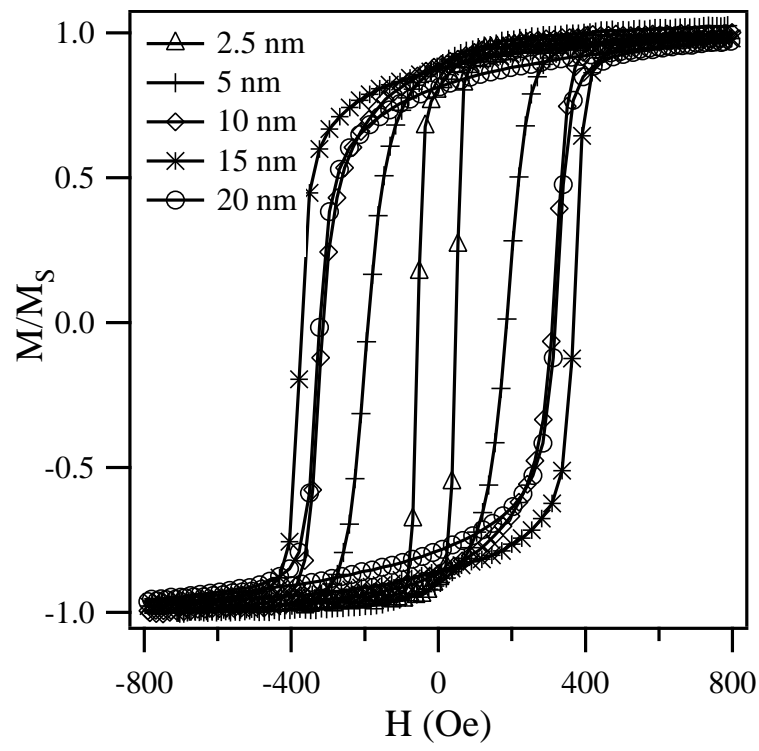

Fig. 1. MOKE longitudinal loops collected on FeCo samples having thickness ranging from 2.5 up to $20 \mathrm{~nm}$.

equipped with a stitching system that allows scanning over an area as wide as $10 \times 10 \mathrm{~cm}^{2}$.

\section{Results and Discussion}

The MOKE hysteresis loops recorded for $2.5 \mathrm{~nm} \leq t \leq 20$ $\mathrm{nm}$ are presented in Fig. 1. As a function of $t$, the shape of the loops does not undergo remarkable modifications, but the value of the coercive field $\left(H_{C}\right)$ increases with $t$ up to $t$ $=15 \mathrm{~nm}$. The SQUID magnetization loops for the samples with $t=50 \mathrm{~nm}$ and $t=100 \mathrm{~nm}$ are shown in Fig. 2(a). In this case, with respect to the thinner samples an increase of saturation field is observed. This change is confirmed by MOKE data, as well. The $\mathrm{H}_{C}$ values as a function of $t$ are presented in Fig. 3. The observed $H_{C}(t)$ dependence is in agreement with the predictions of the random anisotropy model; in particular, the initial increase may be due to an increase in the FeCo nanograin size that may be observed as $t$ changes from $2.5 \mathrm{~nm}$ up to $15 \mathrm{~nm}$. For this thickness, the nanograin size is possibly comparable with the exchange length, so no further increase of $H_{C}$ is observed [6]. Diffraction data support this hypothesis: the samples display a polycrystalline structure and the average grain size deduced with the Scherrer formula [7] increases with $t$; in detail, for the sample with $t=50 \mathrm{~nm}$ the average size is about $15 \mathrm{~nm}$, close to the expected FeCo exchange length, that is about $18.4 \mathrm{~nm}$ [6]. For what concerns the saturation magnetization $\left(M_{S}\right)$ value, it changes slightly $(\sim 10 / 12 \%)$ with $t$, and does not show an identifiable dependence on $t$ (see the inset of Fig. 3). The average $M_{S}$ value is about $1.4 \cdot 10^{6} \mathrm{~A} / \mathrm{m}$, lower than that expected for FeCo samples [8]. This result is in agreement with the $M_{S}$ values obtained from the MOKE and SQUID saturation fields detected with $H$ perpendicular to samples surface; indeed, using this configuration, the saturation field value is equal to $M_{S}$. Those low $M_{S}$ values are possibly ascribable to the presence of a disordered crystalline structure, as evidenced by diffraction measurements. As $M_{S}$ is nearly independent of $t$, the small $M_{S}$ values should not be

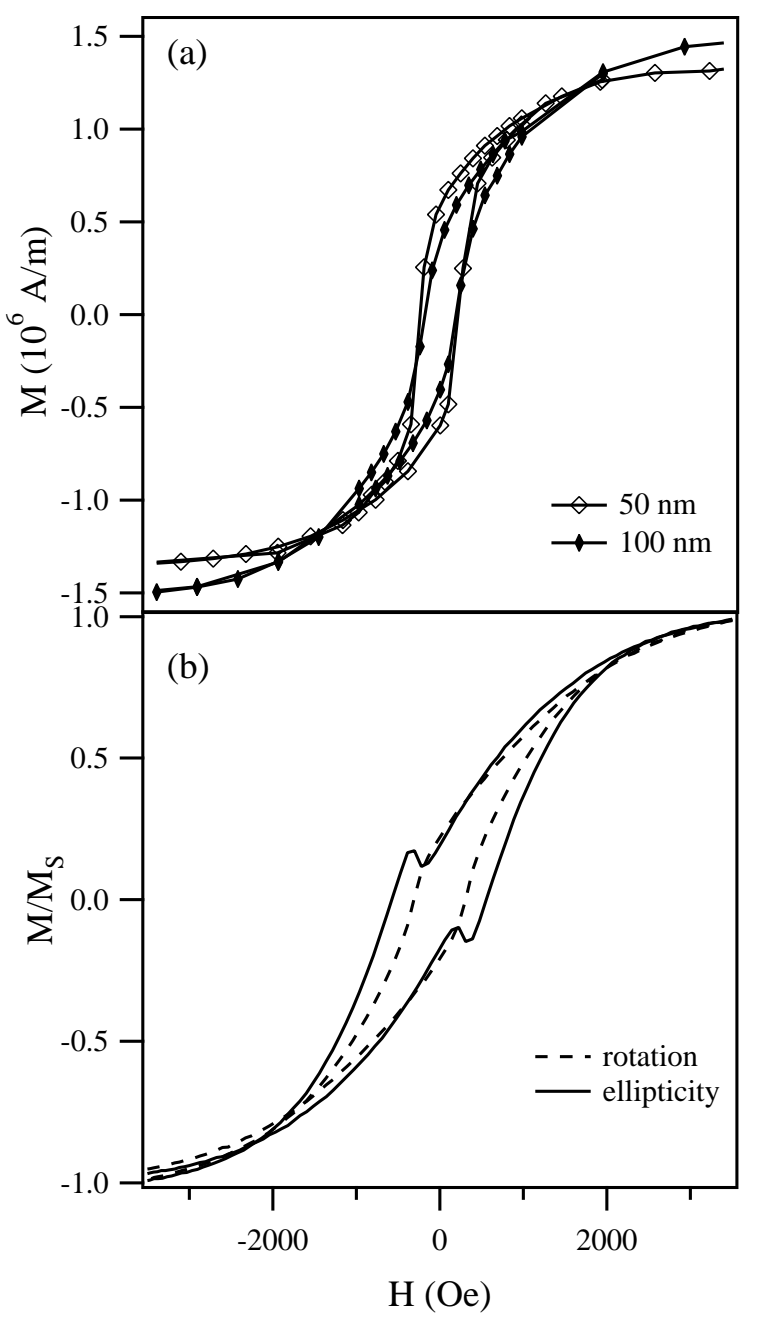

Fig. 2. (a) SQUID magnetization loops recorded on $50 \mathrm{~nm}$ and $100 \mathrm{~nm}$ thick samples; (b) MOKE loops collected in longitudinal configuration on the $50 \mathrm{~nm}$ thick sample showing the ellipticity and rotation dependence on external field. SQUID and MOKE data were measured with $H$ parallel to samples surface.

due to oxidation effects. Besides the increase in the saturation field values, measured with the field applied in the film plane, as $t$ increases, is observed the presence of a two-component magnetic behaviour. In Fig. 2(b), the rotation and ellipticity dependence on $H$ for the $50 \mathrm{~nm}$ thick sample is shown. The two parameters display a different dependence on $H$, and this fact may feature the presence of a non homogeneous magnetic behavior or of second-order magneto-optic effects [9]. Indeed, when a magnetically homogeneous material is considered, rotation and ellipticity are expected to be proportional to $M$, so their normalized dependence on $H$ should overlap. MOKE loops recorded using the polar configuration show that, for $t$ smaller than $20 \mathrm{~nm}$, the $M$ dependence on external field is linear, as expected for measurements performed with $H$ aligned with an hard axis. For $t>20 \mathrm{~nm}$, the loops deviate from linearity and an hysteretic behavior appears.

Finally, the magnetic behavior of the samples was investigated also with MR measurements. For $t<10 \mathrm{~nm}$, the MR signal is very weak, and when $t=10 \mathrm{~nm}$ a contribution of about $0.01 \%$ is observed. For $t=20 \mathrm{~nm}$ the MR signal is more detectable, as showed in Fig. 4; in particu- 


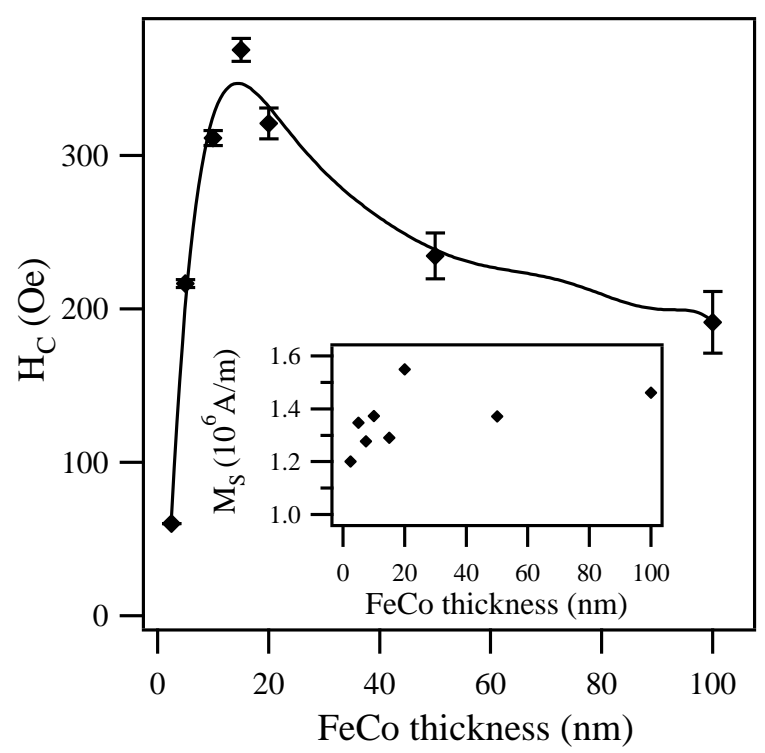

Fig. 3. Coercive field dependence on FeCo film thickness, obtained from MOKE loops. The straight line is just a guide to the eye. In the inset: $M_{S}$ dependence on FeCo thickness, the error bars are comparable to the size of the markers.

lar, it shows a clear correlation with the $H$ dependence of the transverse component of $M$ (Fig. 5). The loop shape is nearly indipendent of $t$; the $M_{y} / M_{s}$ magnitude is higher in the sample with $t=2.5 \mathrm{~nm}$ and decreases with increasing $t$. The MR changes sign as the current-field relative orientation is changed, suggesting the presence of an anisotropic response, as expected for a homogeneous ferromagnetic film. For $t=50 \mathrm{~nm}$, the MR response is nearly isotropic (Fig. 6); just a small anisotropic contribution is found for low values of the external field. This term appears in the same $H$ range where the magnetization loops of this sample displays the hysteretic behavior, whilst the isotropic contribution is displayed in the $H$ range where the slow approach to saturation is observed. This could confirm the hypothesis that, for $t>20 \mathrm{~nm}$, the FeCo films show a behavior that can be ascribed to the presence of two different magnetic components as seen above in the SQUID measurements. The component that shows an hysteretic behavior seems to recall the magnetic properties of the FeCo films with a smaller thickness. The other one, shows a high saturation field and is related to the isotropic MR contribution.

Due to the magnetoelastic properties of FeCo films, to detect possible contributions to their magnetization properties ascribable to the presence of growth-induced stress, we measured the substrate curvature variation induced by the FeCo film for different values of $t$. If we look at the substrate from the FeCo film side, for all the samples the effect of the film deposition is to make the substrate more convex and the change in substrate curvature increases with $t$. The observed increase in substrate convexity can be possibly ascribed to the presence of an intrinsic compressive stress in the FeCo layers. Indeed, when sputtering deposition is considered, the deposited film may develop an intrinsic stress, that can be compressive due to the atomic peening process [5]. In this case, as the FeCo magnetostrictive constants are positive [10], the presence of an in-plane

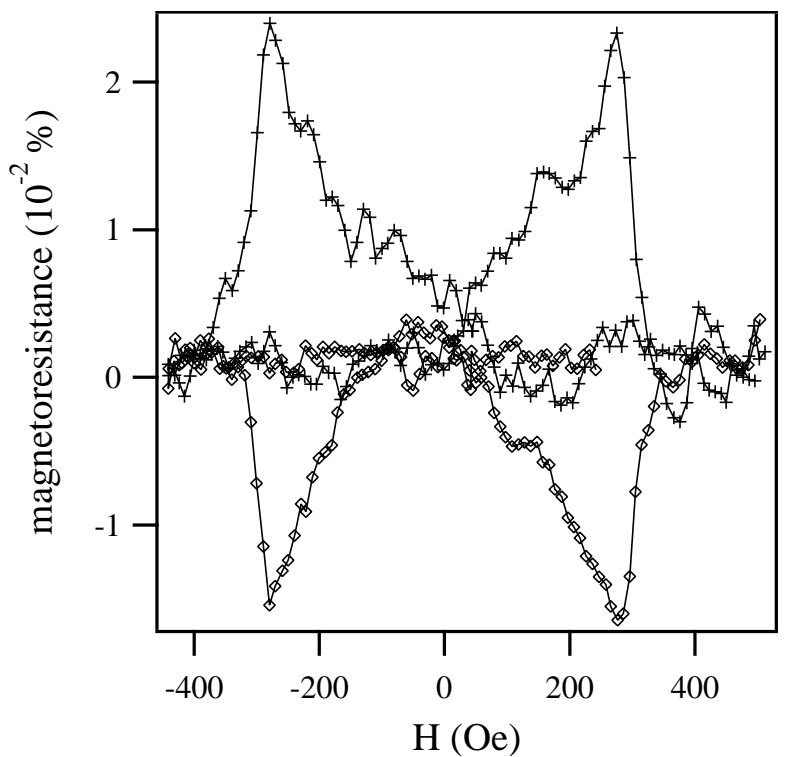

Fig. 4. Magnetoresistance data collected on the $20 \mathrm{~nm}$ thick FeCo sample in two different configurations: current parallel $(\diamond)$ and perpendicular $(+)$ to the magnetic field.

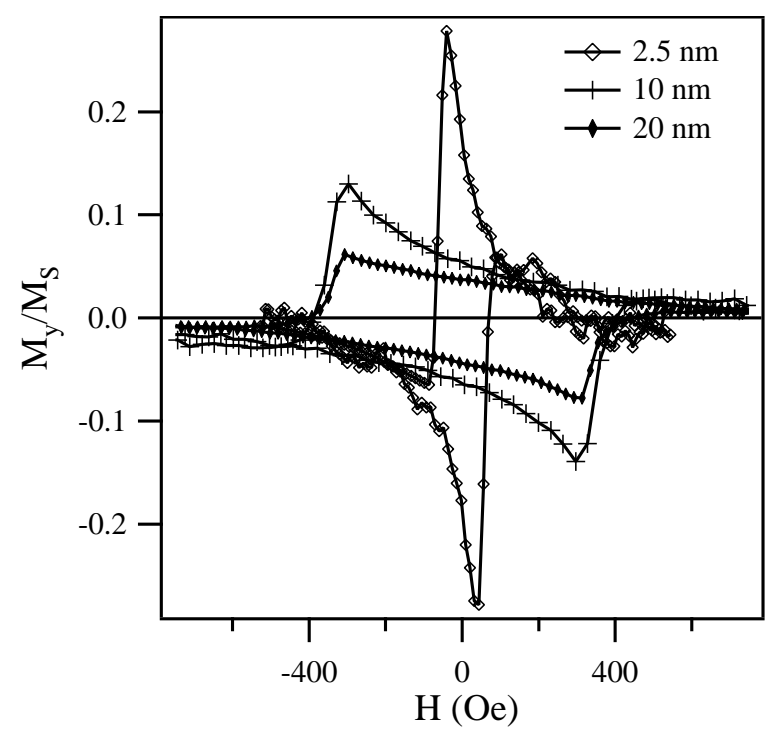

Fig. 5. Dependence of the transverse component of $M\left(M_{y}\right)$, normalized to $M_{S}$, on magnetic filed for different FeCo thickness.

compressive stress may favour the development of an outof-plane easy axis of magnetization [11].

To estimate the stress in the plane of the film $\left(\sigma_{f}\right)$ it is possible to use the Stoney equation [12]:

$$
\sigma_{f}=\frac{E_{s}}{6\left(1-v_{s}\right) t} t_{s}^{2}\left(C-C_{0}\right)
$$

where $t$ is the film thickness, $E_{s}$ is the Young's modulus of the substrate, $v_{s}$ is the Poisson ratio of the substrate, $t_{s}$ is the substrate thickness, $C$ is the curvature measured after the film deposition and $C_{0}$ is the initial curvature. When $t$ $=10 \mathrm{~nm}, \sigma_{f} \sim-600 \mathrm{MPa}$; taking into account the values of FeCo magnetostrictive constants [10] and the fact that the films are polycrystalline [11], this corresponds to an out-of-plane anisotropy constant, $K_{\sigma}$, whose value is equal to $6.7 \cdot 10^{4} \mathrm{~J} / \mathrm{m}^{3}$. If compared to the shape anisotropy 


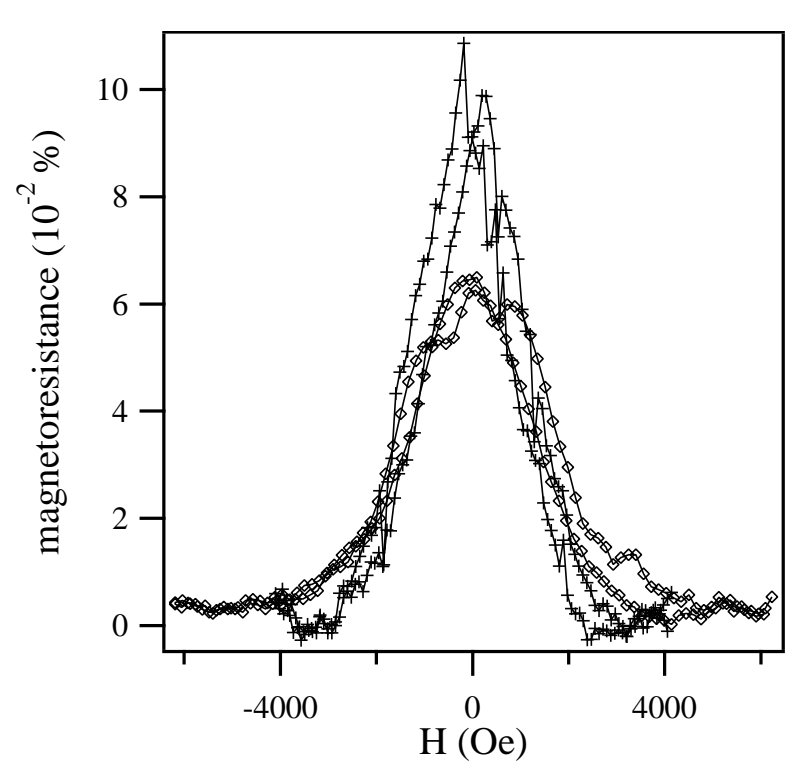

Fig. 6. Magnetoresistance data collected on the $50 \mathrm{~nm}$ thick FeCo sample: current parallel $(\diamond)$ and perpendicular $(+)$ to the magnetic field.

constant, $K_{s} \sim-13 \cdot 10^{5} \mathrm{~J} / \mathrm{m}^{3}$, we have that $\left|K_{s}\right|>K_{\sigma}$, so the shape anisotropy overcomes the magnetoelastic contribution and the samples magnetization preferentially lies in the plane of the film. As $t$ increases, the change in curvature increases and eventually the $K_{\sigma}$ value overcomes the $K_{s}$ one. This is in agreement with the results obtained with magnetic measurements, where as $t$ increases the magnetic behavior of the samples features a progressive switch of the out-of-plane direction from a hard to an easy magnetization axis. A similar effect, for $100 \mathrm{~nm}$ thick films, was observed in FeCo samples produced by fs-pulsed laser deposition; in this case, the compressive in-plane stress was induced thanks to a high substrate temperature during deposition and to the different film and substrate lattice thermal expansion coefficients [13]. The fact that, in our samples, the magnetic and magnetoresistive behaviour of thicker samples is not homogeneous (see. Fig. 2(b) and Fig. 6) is possibly due to the fact that, maybe due to the polycrystallinity of the sample, the stress, and hence the magnetic behavior, is not uniform all over the samples.

\section{Conclusions}

In summary, we studied the magnetic properties of $\mathrm{FeCo}$ polycrystalline thin films with a thickness ranging from $2.5 \mathrm{~nm}$ up to $100 \mathrm{~nm}$. In the first range $(2.5 \mathrm{~nm} \leq t \leq 20$ $\mathrm{nm}$ ) the shape of the films magnetization loops is nearly squared and the coercivity rapidly increases for $t<15$ $\mathrm{nm}$, then it smoothly decreases with $t$. The magnetoresistance measurements show the presence of an anisotropic contribution. All those data confirm that the ferromagnetic films are homogeneous and that the out-of-plane direction is a hard axis. In the second range $(t>20 \mathrm{~nm})$ the shape of the films magnetization loops is no more squared, a large coercivity is still found but remanence decreases and the saturation field increases. The magnetoresistance data indicate that the samples show a non-homogeneous magnetic behavior, as anisotropic MR sources coexist with isotropic MR sources; in-plane and out-of-plane MOKE loops support the presence of magnetic inhomogeneities and confirm that, in this range of $t$, effects ascribable to the progressive development of an out-of-plane magnetization easy-axis are found. These findings are in agreement with the measured change in substrate curvature induced by the FeCo films, that features the presence of a compressive in-plane stress within the films and a positive out-ofplane anisotropy constant, accordingly. This intrinsic stress increases with $t$, and finally becomes comparable to the shape anisotropy contribution, thus promoting the presence of an out-of-plane easy axis. Possibly due to the polycrystalline structure of the samples, the value of the out-ofplane anisotropy constant is not uniform throughout the samples, thus explaining the inhomogeneous magnetic behavior displayed by MOKE and MR measurements.

This research work was in part sponsored by MIUR under project FIRB2010 'Tailoring the magnetic anisotropy of nanostructures for enhancing the magnetic stability of magnetoresistive devices - NANOREST'

\section{References}

1. C. L. Platt, A. E. Berkowitz, D. J. Smith, M.R. McCartney, J. Appl. Phys. 88, (2000) 2058

2. N. Miyamoto, H. Ohmori, K. Mamiya, S. Nakagawa, J. Appl. Phys. 107, (2010) 09CT19

3. W. Yu, J. A. Brian, W. C. Uhlig, J. Unguris, J. Appl. Phys. 99, (2006) 08B706

4. S. Dokupil, M.-T. Bootsmann, S. Stein, M. Löhndorf, E. Quandt, J. Magn. Magn. Mater. 290-291, (2005) 795

5. R. Koch, Surf. \& Coat. Technol. 204, (2010) 1973

6. M. Vopsaroiu, M. Georgieva, P. J. Grundy, G. Vallejo Fernandez, S. Manzoor, M. J. Thwaites, K. O'Grady, J. Appl. Phys. 97, (2005) 10N303

7. A. L. Patterson, Phys. Rev. 56, (1939) 978

8. A. Dìaz-Ortiz, R. Drautz, M. Fähnle, H. Dosch, J. Sanchez, Phys. Rev. B. 73, (2006) 224208

9. R.M. Osgood III, S.D. Bader, B.M. Clemens, R.L. White, H. Matsuyama; J. Magn. Magn. Mater. 182, (1998) 297

10. J. McCord, S. Dieter, K. Seemann, J. Magn. Magn. Mater. 271, (2004) 46

11. P. Zou, W. Yu, J. A. Bain, J. Appl. Phys. 91, (2002) 7830

12. R. W. Hoffman, in G. Hass R. E. Thun (Eds.), Physics of Thin Films, vol. 3 (Accademic Press, New York, 1966) 211

13. G. Ausanio, V. Iannotti, C. L. Hison, L. Lanotte, S. Amoruso, C. Aruta, X. Wang, M. Tamisari, Thin Solid Films 519, (2011) 5420 